\title{
COMPARAÇÃO DE MÉTODOS PARA ESTIMATIVA DO PLASTOCRONO EM ALGODOEIRO EM CONDIÇÕES TROPICAIS ${ }^{1}$
}

\author{
Luiz Carlos Pereira², José Holanda Campelo Júnior ${ }^{2}$, Alessandro Ferronato ${ }^{2}$
}

\section{ABSTRACT \\ COMPARISON OF METHODS FOR ESTIMATING \\ PLASTOCHRON IN COTTON PLANT UNDER TROPICAL WEATHER CONDITIONS}

The time for growth and development of plants is influenced by air temperature and other environmental variables. This research was based on three methods for calculating plastochron timing of cotton plant under tropical weather conditions, using thermal units accumulation as variable, calculated from the average daily air temperature, considering the concepts of degrees-day and accumulated thermal time. Two methods for calculating average daily temperature and three methods for obtaining Degrees-Day values and accumulated thermal time were used. Whereas the accumulated thermal time is a method that considers that the growth and development of plants have linear response to the air temperature influence, it was also tested a non-linear approach, as described by Wang \& Engel (1998), called, in this study, $\beta$ function. For the $\beta$ function, average daytime temperatures were also used, obtained by the two different calculation methods. The $\beta$ function was the best option for estimating plastochron and the method for calculating the Average Daily Temperature influenced the results.

KEY-WORDS: Gossypium hirsutum; degrees-day; beta function; physiological age; thermal units.

\section{INTRODUÇÃO}

O algodão é um dos produtos agrícolas mais importantes no mundo e, consequentemente, o seu cultivo tem lugar de destaque no cenário do Agronegócio. No Brasil, não é diferente e o Estado de Mato Grosso é, na atualidade, o principal produtor desta fibra. Este fato tem gerado demandas para os pesquisadores, em diversas áreas das agrociências.

Dentre estas demandas, o estudo de aspectos fenológicos das plantas é de fundamental importância, pois representa variáveis que podem ser utilizadas na previsão de eventos importantes

\section{RESUMO}

O tempo de crescimento e desenvolvimento de plantas é influenciado pela temperatura média do ar, entre outras variáveis ambientais. Neste trabalho, foram utilizados três métodos de cálculo para contagem do tempo do plastocrono em algodoeiro, em condições de clima tropical, utilizando-se, como variável, o acúmulo de unidades térmicas, calculadas a partir da temperatura média diária do ar, considerando-se os conceitos de graus-dia e soma térmica acumulada. Foram utilizados dois métodos de cálculo da Temperatura Média Diária e três métodos de obtenção dos valores de Graus-Dia e Soma Térmica Acumulada. Considerando-se que a Soma Térmica Acumulada é um método que considera que o crescimento e desenvolvimento das plantas apresentam resposta linear à influência da temperatura do ar, foi testada, também, uma abordagem não-linear, descrita por Wang \& Engel (1998), utilizada, neste trabalho, com a denominação de Função $\beta$. Na Função $\beta$, também foram utilizadas as Temperaturas Médias Diárias obtidas pelos dois diferentes métodos de cálculo. Os resultados obtidos permitiram considerar que a Função $\beta$ foi melhor na estimativa do plastocrono e que o método de cálculo da Temperatura Média Diária influenciou os resultados obtidos.

PALAVRAS-CHAVE: Gossypium hirsutum; graus-dia; função beta; idade fisiológica; unidades térmicas.

na tomada de decisão sobre o manejo da cultura em condições de campo. Khan (2003) trabalhou com a contagem de nós na haste principal do algodoeiro, por considerar esta variável importante, entre outras coisas, para ajustamento das estações de crescimento do algodoeiro. O plastocrono, que pode ser definido como o tempo necessário para o aparecimento de um novo nó na planta, considerado, neste trabalho, como um novo nó na haste principal do algodoeiro, representa importância em modelos de simulação matemática do crescimento do algodoeiro, contribuindo para o aprimoramento de sistemas deste tipo. 
A temperatura do ar influencia, diretamente, a maioria dos parâmetros fenológicos, dentre eles o plastocrono, fato que tem motivado a realização de inúmeros trabalhos sobre este fenômeno, utilizandose diversos métodos para a mensuração e estimativa desta influência.

Streck et al. (2005) trabalharam com vários métodos de cálculo da Soma Térmica Acumulada, para estimar o tempo fisiológico decorrido entre a emissão de dois nós, na haste principal de meloeiro. Sentelhas et al. (1994) mensuraram o desenvolvimento de cultivares de girassol, nos subperíodos emergência-floração e floração-maturação, por meio de soma térmica acumulada. Infeld et al. (1998) estimaram, em plantas de arroz, a duração do período vegetativo, por meio de soma térmica. Em várias regiões do Estado de São Paulo, Barbano et al. (2002) estimaram a duração do ciclo de desenvolvimento de ervilhas, sob influência da temperatura do ar.

Em todos os trabalhos citados, foram utilizados métodos de cálculo que consideram uma ótica de resposta linear dos fenômenos de crescimento, à temperatura do ar.

Estes cálculos são realizados a partir da obtenção dos valores de graus-dia, que podem ser definidos como cada grau de temperatura acima de uma temperatura basal inferior, abaixo da qual a espécie não apresentaria desenvolvimento, e de soma térmica, definida como o acúmulo de graus-dia necessários para se completar um ciclo, processo ou subperíodo do desenvolvimento de uma espécie vegetal.

Os modelos de cálculo dos graus-dia podem ser muito simples, envolvendo apenas uma temperatura basal inferior e temperaturas máxima e mínima do dia. Em muitos casos, não têm havido diferenças entre estes modelos mais simples e outros mais complexos, que incluem uma temperatura ótima e uma basal superior.

Arnold (1959) encontrou entre 2\% e 4\% de diferenças, ao comparar resultados obtidos por modelos destes dois tipos. Viator et al. (2005) relataram que, em trabalhos realizados na região oeste do CottonBelt americano, foi encontrado um incremento de acurácia, com a introdução de valores cardinais mais complexos aos modelos, provavelmente devido à maior amplitude de temperatura do ar naquela região. No entanto, a base teórica destes métodos considera que a resposta das plantas ao efeito da temperatura seja linear, conforme preconizado por Arnold (1959).
Viator et al. (2005), trabalhando com tempo de maturação das maçãs do algodoeiro, evidenciaram ajuste satisfatório para os dados, utilizando soma térmica obtida com temperatura basal de $15,5^{\circ} \mathrm{C}$. Relataram, ainda, que graus-dia têm sido utilizados para predição do desenvolvimento de culturas e ajudado, entre outras coisas, na seleção de variedades e no tempo de irrigação. No cálculo dos graus-dia, pode ser, ainda, considerada uma temperatura basal superior, acima da qual não há desenvolvimento da cultura.

O trabalho de Wang \& Engel (1998) apresentou uma ótica diferente, considerando que a resposta fenológica das plantas ao efeito da temperatura é nãolinear. No modelo proposto por Wang \& Engel (1998), a taxa diária de desenvolvimento das plantas $(r)$ processo ou subperíodo do seu desenvolvimento - é igual à taxa máxima de desenvolvimento $\left(r_{\max }\right)$ da planta - processo ou subperíodo do seu desenvolvimento - multiplicada por uma função não-linear da temperatura $(f(T))$, conforme $r=r_{\text {max }} \cdot f(T)$.

O cálculo de $f(T)$ exige o conhecimento das temperaturas Cardinais, Basal Inferior, Basal Superior e Temperatura Ótima. A temperatura Basal Inferior pode ser definida como a temperatura abaixo da qual não existe crescimento ou desenvolvimento da espécie em estudo; a Basal Superior é aquela acima da qual o desenvolvimento é nulo; e a Temperatura Ótima é aquela em que o crescimento e desenvolvimento são máximos.

O crescimento da planta, ou desenvolvimento do processo em estudo, se completa quando o somatório das taxas diárias de crescimento/desenvolvimento (r) atinge o valor 1,0:

$$
\left(\sum_{m=1}^{n} r=1\right)
$$

Alguns estudos, como o de Wang \& Engel (1998), com trigo, e Campelo Júnior et al. (2005), com mangabeira, simularam o desenvolvimento fenológico, utilizando este modelo.

O objetivo deste trabalho foi comparar os resultados obtidos na estimativa do plastocrono em algodoeiro, a partir da soma térmica acumulada, que considera que o desenvolvimento das plantas responde, de forma linear, à influência da temperatura, e com o método descrito por Wang \& Engel (1998), que considera resposta não-linear das plantas a esta variável, considerando-se, ainda, duas formas de obtenção da média diária da temperatura. 


\section{MATERIAL E MÉTODOS}

Os dados utilizados neste trabalho foram coletados em dois experimentos. O Experimento 1 foi instalado na Fazenda Experimental da Universidade Federal do Mato Grosso, localizada em Santo Antônio do Leverger (MT), nas coordenadas $15,9^{\circ} \mathrm{S}$ e $54^{\circ} \mathrm{W}$, à altitude de $140 \mathrm{~m}$. O Experimento 2 foi instalado no Campo Experimental do Centro Universitário de Várzea Grande (Univag), em Várzea Grande (MT), nas coordenadas $15,6^{\circ} \mathrm{S}$ e $56,1^{\circ} \mathrm{W}$ e altitude de $150 \mathrm{~m}$.

Os dados do Experimento 1 foram coletados em 6 lisímetros de nível de lençol freático constante, os quais faziam parte de um experimento instalado em área de $20 \mathrm{~m} \times 30 \mathrm{~m}$, pertencente à Fazenda Experimental da Universidade Federal do Mato Grosso.

Cada caixa lisimétrica, medindo 1,2 $\mathrm{m} \times 1,0 \mathrm{~m}$, foi considerada uma parcela experimental, sendo que, em cada caixa, foram medidas 3 plantas, escolhidas aleatoriamente. Os intervalos de tempo entre as medidas variaram de 5 a 17 dias. As coletas foram realizadas de 22 de abril a 05 de agosto de 2006 . Foi utilizada adubação de plantio com $30 \mathrm{~kg} / \mathrm{ha}$ de $\mathrm{N}$, $80 \mathrm{~kg} / \mathrm{ha}$ de $\mathrm{P}_{2} \mathrm{O}_{5}$ e $30 \mathrm{~kg} /$ ha de $\mathrm{K}_{2} \mathrm{O}$. Aos 80 dias, foi realizada cobertura com $30 \mathrm{~kg} / \mathrm{ha}$ de $\mathrm{N}$ e $30 \mathrm{~kg} / \mathrm{ha}$ de $\mathrm{K}_{2} \mathrm{O}$. O espaçamento utilizado foi de $1,0 \mathrm{~m}$ entre linhas, sendo utilizada população adensada com número médio de 17 plantas por metro. Como as parcelas eram caixas lisimétricas de lençol freático constante, considerou-se que não houve déficit hídrico durante o período de coleta de dados no experimento.

O Experimento 2 foi conduzido em parcela com medidas de $10 \mathrm{~m}$ x $10 \mathrm{~m}$, com adubação de plantio e uma cobertura, aos 50 dias, nas mesmas doses aplicadas ao Experimento 1. Este experimento recebeu irrigação suplementar suficiente para manter a umidade do solo em valores próximos à capacidade de campo, considerando-se, portanto, que não houve déficit hídrico durante as medidas realizadas. As coletas foram realizadas de 05/09/2006 a 06/10/2006, com intervalos variando entre 2 e 7 dias.

Para o presente trabalho, foi realizada a contagem dos nós na haste principal, a cada coleta realizada. Os dados de temperatura do ar foram obtidos na Estação Meteorológica Padre Ricardo Remeter, localizada a cerca de $1,5 \mathrm{~km}$ do local das parcelas do Experimento 1 e a cerca de $20 \mathrm{~km}$ da parcela do Experimento 2.
As variáveis utilizadas para temperatura média diária foram a Temperatura Média Simples $(T M S)$ e a Temperatura Média Compensada (TMc), obtidas, respectivamente, pelas equações

$$
T M S=\frac{T_{x}+T_{n}}{2},
$$

onde $T_{x}$ e $T_{n}$, são, respectivamente, as temperaturas máxima e mínima do ar, e

$$
T M c=\frac{T_{12}+T_{x}+T_{n}+\left(2 T_{24}\right)}{5},
$$

onde TMc, $T_{12}, T_{24}, T_{\mathrm{x}}$ e $T_{\mathrm{n}}$ são, respectivamente, as temperaturas, média, temperatura às 12 horas, temperatura às 24 horas, temperatura máxima e mínima do ar. As horas de leitura das temperaturas são referidas ao observatório de Greenwich.

Este trabalho utilizou duas abordagens para a contagem do tempo fisiológico da cultura. A primeira considera que o tempo fisiológico é uma função linear do acúmulo de unidades térmicas, ou grausdia, que foram chamados de soma térmica diária $(S T d)$ e calculados por três métodos, utilizados por Gilmore \& Rogers (1958) e Arnold (1959): Método 1: $S t_{d}=\left(T_{m}-T b\right)$, considerando $T_{m}=T b$, se $T_{m}<T b$; Método 2: $S t_{d}=\left(T_{m}-T b\right)$, considerando $T_{m}=T b$, se $T_{m}<T b$, e $T_{m}=T o$, se $T_{m}>T o$; e Método 3: $S t_{d}=$ $\left(T_{m}-T b\right)$, caso $T b<T_{m} \leq T_{0}$, e, caso $T o<T_{m} \leq T_{x}$,

$$
S t_{d}=\frac{(T o-T b)\left(T_{x}-T_{m}\right)}{\left(T_{x}-T o\right)} .
$$

O somatório destes graus-dia, ao longo do tempo, foi acumulado nas Somas Térmicas Acumuladas, que foram denominadas, neste trabalho, como $S T a S$ e $S T a C$, conforme tenham sido obtidas pelo acúmulo de graus-dia calculados com TMS ou TMc, respectivamente.

Além destes métodos, foi utilizado um método cuja abordagem de acúmulo de unidades térmicas determina o crescimento e desenvolvimento da cultura como função não-linear da temperatura. Este método foi proposto por Wang \& Engel (1998) e chamado, neste trabalho, Função $\beta, r=r_{\text {max }} \cdot f(T)$, onde $r$ é a taxa diária de desenvolvimento vegetal, $r_{\max }$ a taxa diária máxima de desenvolvimento vegetal e $f(T)$ uma função da temperatura, obtida a partir da equação

$$
f(T)=\frac{2\left(T_{m}-T b\right)^{\alpha}(T o-T b)^{\alpha}\left(T_{m}-T b\right)^{2 \alpha}}{(T o-T b)^{2 \alpha}},
$$


onde $T$ é a temperatura média do ar, $T b$ a temperatura basal inferior e $\alpha$ um parâmetro da equação calculada em função das temperaturas cardinais, obtido por meio da equação

$$
\alpha=\frac{\ln 2}{\ln \frac{T B-T b}{T o-T b}},
$$

que considera que, quando $T \leq T b$ ou $T \geq T B, f(T)=0$.

As temperaturas cardinais utilizadas foram 14, 30 e 35, respectivamente, para Temperatura Basal Inferior ( $T b)$, Temperatura Ótima (To) e Temperatura Basal Superior $(T B)$, conforme Azevedo et al. (2004).

O plastocrono foi obtido por meio do inverso do coeficiente angular da reta de regressão linear entre o número de nós acumulados na haste principal e o valor da soma térmica acumulada, quando utilizada a Soma Térmica Acumulada.

Quando foi utilizada a Função $\beta$, o surgimento de um novo nó na haste principal ocorreu a cada vez que o somatório das taxas diárias de desenvolvimento $(r)$ atingiu o valor 1 , conforme a equação

$$
\sum_{m=1}^{n} r_{m}=1
$$

onde $r$ é a taxa diária de desenvolvimento e $m$ a contagem de dias desde o início de cada período do ciclo em estudo.

Os dados do Experimento 1 foram utilizados para ajustar os dados medidos, obtendo-se os valores médios de plastocrono, e os dados do Experimento 2 utilizados para aferir o desempenho dos modelos. A acurácia das estimativas foi medida por dois índices, conhecidos como Índice de Concordância de
Willmott (d), descrito em Willmott et al. (1985), e Coeficiente "c", conforme descrições encontradas em Camargo \& Sentelhas (1997) e Andrade Júnior et al. (2003).

\section{RESULTADOS E DISCUSSÃO}

Na Tabela 1, estão os dados observados para o surgimento dos nós na haste principal do algodoeiro. Verifica-se que o coeficiente de variação ficou abaixo de $10 \%$, para a maioria das coletas, valores, estes, da mesma ordem de grandeza dos valores encontrados por Viator et al. (2005), em trabalho sobre o tempo de maturação da maçã do algodoeiro, no qual os valores de CV variaram entre 11,50 e 13,07. As maiores variações observadas, no início e no final do período de avaliação, provavelmente, estiveram relacionadas a diferenças na germinação das sementes e emergência das plântulas na implantação do experimento, e com a perda de algumas medidas ocorridas no último período de coleta apresentado.

Na Figura 1, estão os dados de temperatura média obtidos no período de observação da cultura estudada. Observa-se que os valores oscilaram entre $29,08^{\circ} \mathrm{C}$ e $17,24^{\circ} \mathrm{C}$, para $T M c$, e entre $29,00^{\circ} \mathrm{C}$ e $17,70^{\circ} \mathrm{C}$, para $T M s$. Os valores de temperatura média mantiveram estabilidade em torno $\operatorname{dos} 25^{\circ} \mathrm{C}$, na maior parte do tempo de observação, aproximadamente entre os 35 DAE e 85 DAE. Neste período, foi observada diferença entre os valores obtidos pelos dois métodos de cálculo utilizados. Esta diferença esteve associada à maior influência da temperatura noturna na obtenção da média nos períodos em que as temperaturas estiveram mais estáveis.

Tabela 1. Número médio de nós, por planta, acumulados na haste principal do algodoeiro, contados ao longo do período do experimento (Santo Antônio do Leverger, MT, 2006).

\begin{tabular}{ccccccccc}
\hline \multirow{2}{*}{ Parcelas } & \multicolumn{8}{c}{ Dias Após a Emergência (DAE) } \\
\cline { 2 - 9 } & 17 & 24 & 33 & 45 & 59 & 77 & 101 & 108 \\
\hline 1 & 3,3 & 5,7 & 8,7 & 11,3 & 13,0 & 14,3 & - & - \\
2 & 4,0 & 5,7 & 7,7 & 11,3 & 13,7 & 14,0 & 16,7 & 17,3 \\
3 & 3,7 & 5,7 & 8,3 & 10,0 & 12,0 & 13,7 & 17,3 & 21,0 \\
4 & 3,0 & 5,0 & 7,0 & 9,7 & 12,7 & 14,0 & 17,3 & 17,7 \\
5 & 3,0 & 5,3 & 7,3 & 9,3 & 11,7 & 13,7 & 15,3 & - \\
6 & 3,3 & 5,3 & 7,7 & - & 13,3 & 15,3 & 19,7 & - \\
\hline Média & 3,39 & 5,44 & 7,78 & 10,33 & 12,72 & 14,17 & 17,27 & 18,67 \\
Sd & 0,39 & 0,27 & 0,62 & 0,94 & 0,77 & 0,62 & 1,57 & 2,03 \\
CV & 11,50 & 5,00 & 7,98 & 9,12 & 6,07 & 4,40 & 9,10 & 10,86 \\
\hline
\end{tabular}


No entanto, nos períodos onde a temperatura apresentou maior variação (5 DAE a 9 DAE, 15 DAE a 30 DAE e 93 DAE a 101 DAE, aproximadamente), pode-se observar que os valores obtidos pelos dois métodos aproximaram-se (Figura 1). Estes resultados demonstram tendência para a obtenção de valores da temperatura média diária diferentes, quando se utilizam diferentes métodos de cálculo, principalmente quando as temperaturas apresentamse mais estáveis. Considerando-se que a maioria dos trabalhos nesta área são realizados na região Sul do País, onde as amplitudes térmicas são maiores, a utilização de métodos de cálculo para as temperaturas médias diárias deve ser observada com cuidado, para utilização em regiões intertropicais, onde as amplitudes térmicas são menores.

Na Tabela 2, estão os dados de soma térmica acumulada, para cada subperíodo considerado neste trabalho. Foram utilizados três métodos de cálculo dos valores de graus-dia. No entanto, os valores encontrados para a soma térmica calculada pelos três métodos foram idênticos.

Isto ocorreu devido ao fato de as médias diárias de temperatura terem flutuado entre os valores de $T b$ e To. Deste modo, a utilização de To e TB acabaram por não influenciar o cálculo dos graus-dia. Streck et al. (2005), trabalhando com o efeito da temperatura na emissão de nós em meloeiro, encontraram diferenças na estimativa do tempo fisiológico, que foram atribuídas à metodologia de cálculo dos graus-dia, o que não foi observado neste trabalho. Esta discordância, provavelmente, esteve associada à amplitude térmica, que, na região intertropical onde foi conduzido o presente experimento, é menor que as encontradas na região Sul do Brasil, onde foi conduzido o experimento desses autores. Por outro lado, foram observadas diferenças, quando utilizados diferentes métodos de cálculo da temperatura média.

Na Tabela 3, estão listados os dados obtidos para o número de graus-dia acumulados para o aparecimento de um novo nó, a partir de STaS e de STaC.

Este resultado era esperado, considerando-se o que foi anteriormente exposto sobre os métodos

Tabela 2. Somas térmicas acumuladas nos subperíodos considerados no experimento, sendo a Soma Térmica Acumulada calculada com TMs (STaS), a Soma Térmica Acumulada calculada com TMc $(S T a C)$ e a contagem cronológica do tempo em Dias Após a Emergência (DAE) (Santo Antônio do Leverger, MT, 2006).

\begin{tabular}{cccc}
\hline Data & $\begin{array}{c}\text { DAE } \\
\text { (dias) }\end{array}$ & $\begin{array}{c}\text { STaS } \\
\text { (Graus-dia) }\end{array}$ & $\begin{array}{c}S T a C \\
\text { (Graus-dia) }\end{array}$ \\
\hline $13 / 05 / 2006$ & 17 & 167,00 & 150,26 \\
$20 / 05 / 2006$ & 24 & 241,85 & 218,50 \\
$29 / 05 / 2006$ & 33 & 326,85 & 295,54 \\
$10 / 06 / 2006$ & 45 & 467,90 & 419,66 \\
$24 / 06 / 2006$ & 59 & 624,75 & 553,44 \\
$12 / 07 / 2006$ & 77 & 825,90 & 731,94 \\
$05 / 08 / 2006$ & 101 & 1071,50 & 948,88 \\
$12 / 08 / 2006$ & 108 & 1151,65 & 1023,54 \\
\hline
\end{tabular}

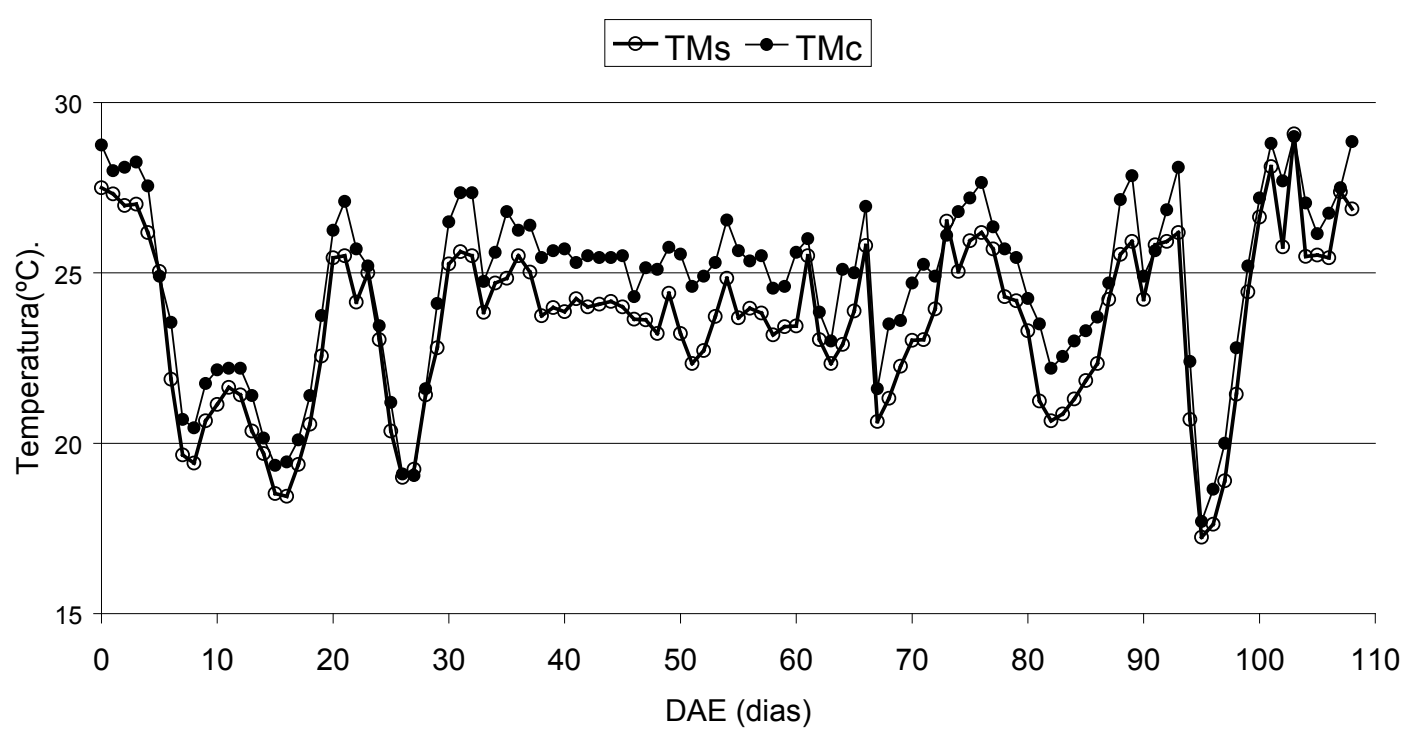

Figura 1. Variação das Temperaturas Médias Diárias, obtidas pelos métodos da Temperatura Média Simples (TMs) e pela Temperatura Média Compensada (TMc), durante o período do experimento (Santo Antônio do Leverger, MT, 2006). 
de obtenção da temperatura média. Deste modo, é recomendável a divulgação das unidades térmicas necessárias para se completar um determinado ciclo vegetal de interesse e o método de cálculo da temperatura média utilizado para sua obtenção.

Ainda na Tabela 3, podem-se observar os resultados obtidos para a contagem do tempo cronológico necessário para o aparecimento de um novo nó. Os modelos apresentaram $r^{2}$ acima de 0,90 , indicando que todos eles ajustaram-se aos dados coletados. Comparando-se o erro padrão apresentado na Tabela 3, é possível observar que o menor valor encontrado foi para o ajuste linear entre DAE e o número de nós.

Na Figura 2, são apresentados os dados obtidos pela aplicação da Função $\beta$. Os dados estimados ajustaram-se aos dados medidos, apresentando $\mathrm{r}^{2} \mathrm{de}$ 0,939 e 0,940 , para as estimativas obtidas com a Função $\beta$, quando consideradas as Temperaturas Médias Diárias calculadas em $T M s$ e $T M c$, respectivamente. O coeficiente angular da reta de ajuste aos dados de $T M c$ foi mais próximo da unidade, significando que a estimativa foi mais próxima do valor medido.

Os resultados obtidos apontam uma necessidade de reavaliação da forma como se utilizam estimativas de eventos fisiológicos, a partir da influência da temperatura do ar, em modelos matemáticos de crescimento. Outros autores já alertaram para a necessidade de se reavaliar a utilização de modelos de cálculo com abordagem linear (Wang \& Engel 1998, Campelo Júnior et al. 2005) e os resultados obtidos neste trabalho corroboram esta visão. Este fato pode representar importantes melhorias em sistemas de simulação matemática do crescimento de culturas agrícolas, tanto do ponto de vista da utilização destas ferramentas para a pesquisa quanto para o processo de tomada de decisão em condições reais de manejo das culturas.

Tabela 3. Valores de tempo cronológico medidos em Dias por nó e tempo fisiológico medido em Graus-Dia por nó (GD/ nó), necessários para o surgimento de um novo nó na haste principal do algodoeiro cultivar Acala 90 (Santo Antônio do Leverger, MT, 2006).

\begin{tabular}{ccccc}
\hline Variáveis & $\begin{array}{c}\text { Coeficiente } \\
\text { angular }\end{array}$ & $\mathrm{R}^{2}$ & $\begin{array}{c}\text { Erro } \\
\text { padrão }\end{array}$ & Plastocrono \\
\hline \multirow{4}{*}{ GD/nó } \\
STaS & 0,0146 & 0,9394 & 1,2577 \\
STaC & 0,0166 & 0,9400 & 1,2518 & 68,45 \\
\cline { 2 - 5 } DAE & 0,1590 & 0,9400 & 1,2509 & 6,29 \\
\cline { 2 - 5 } & & \multicolumn{4}{c}{ Dias/nó } \\
\hline
\end{tabular}

* DAE: Dias Após a Emergência.
Para medir a acurácia dos modelos lineares desenvolvidos para a estimativa do plastocrono, estes foram testados contra os dados medidos no Experimento 2. Na Figura 3, consta o ajuste dos dados Medidos x Estimados, utilizando-se DAE como variável independente.

Pode-se observar que houve ajuste linear entre os dados medidos e estimados. No entanto, observase tendência a uma subestimativa dos dados, visto que o valor do coeficiente angular da reta foi menor que a unidade. Pesquisadores têm sido unânimes na afirmação de que o tempo cronológico não é adequado para a previsão da duração de eventos fenológicos (Arnold 1959, Sentelhas et al. 1994, Infeld et al. 1998, Wang \& Engel 1998, Barbano et al. 2002, Campelo Júnior et al. 2005, Viator et al. 2005).

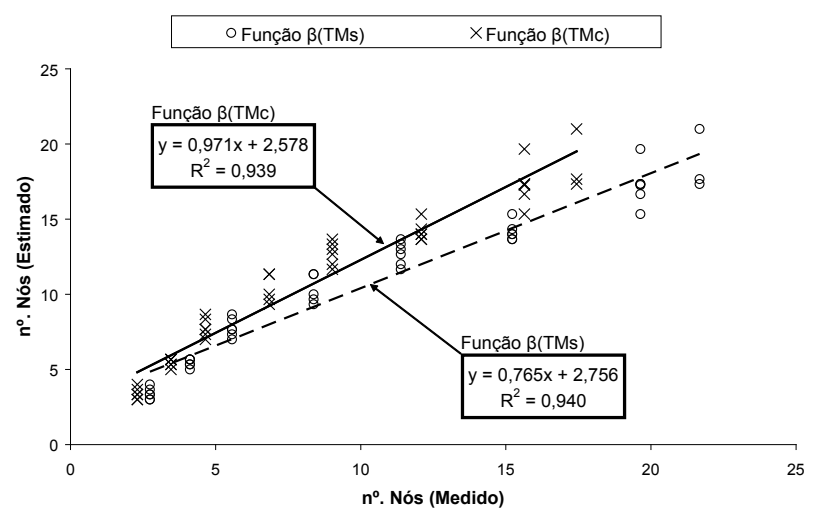

Figura 2. Número de nós acumulados na haste principal do algodoeiro Acala 90, Medido x Estimado, pela Função $\beta$, considerando-se as Temperaturas Médias Diárias TMs e TMc (Santo Antônio do Leverger, MT, 2006).

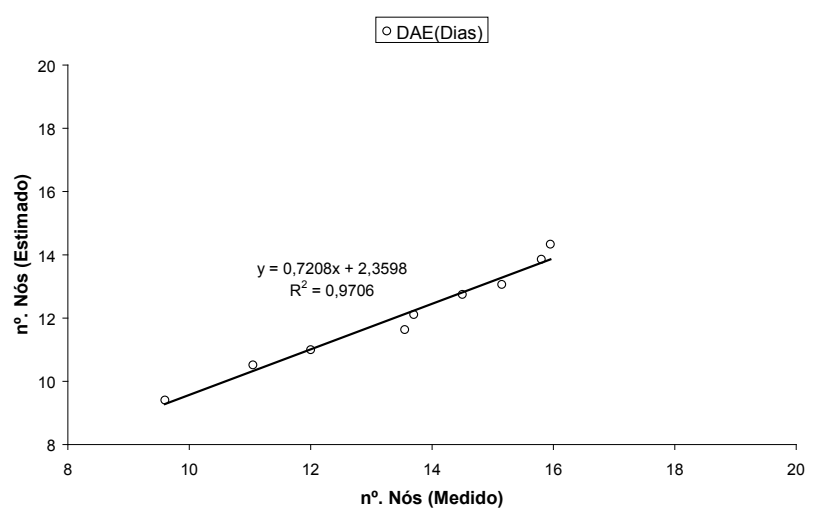

Figura 3. Número de nós acumulados na haste principal do algodoeiro Acala 90, Medido x Estimado, em função do tempo cronológico contado em Dias Após a Emergência (DAE) (Várzea Grande, MT, 2006). 
Na Figura 4, estão demonstrados os ajustes obtidos entre os dados medidos e estimados, a partir do modelo desenvolvido com a soma térmica acumulada. O coeficiente angular da reta foi mais próximo da unidade, evidenciando que as estimativas deste modelo foram melhores, em comparação com as obtidas utilizando-se o DAE.

Os ajustes obtidos utilizando-se a função descrita por Wang \& Engel (1998) foram demonstrados na Figura 5. Considerando-se os coeficientes angulares, observa-se tendência a uma superestimativa dos valores do plastocrono, quando da utilização deste modelo.

Foram utilizados, ainda, dois métodos para mensuração da acurácia das estimativas, propostos

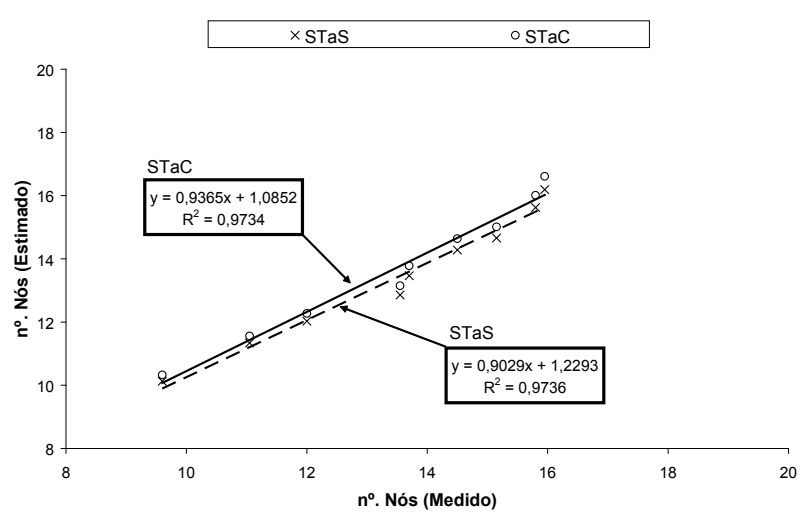

Figura 4. Número de nós acumulados na haste principal do algodoeiro Acala 90, Medido x Estimado, em função das Somas Térmicas Acumuladas, considerandose a Temperatura Média Diária Simples $(S T a S)$ e a Temperatura Média Diária Compensada (STaC) (Várzea Grande, MT, 2006).

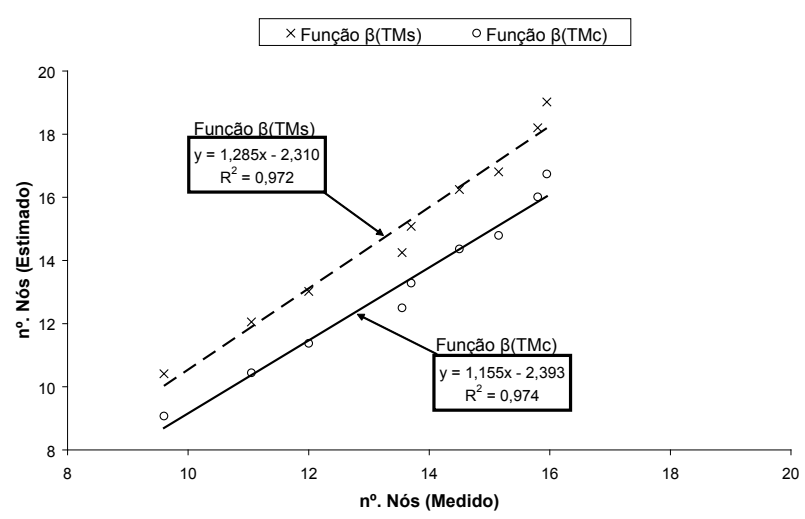

Figura 5. Número de nós acumulados na haste principal do algodoeiro Acala 90, Medido x Estimado, pela Função $\beta$, considerando-se as Temperaturas Médias Diárias TMs e TMc (Várzea Grande, MT, 2006).
Tabela 4. Índice de concordância de Willmot (d) e índice "c", proposto por Camargo \& Sentelhas (1997), para os modelos testados quanto à previsão do plastocrono em algodoeiro (Santo Antônio do Leverger, MT, 2006).

\begin{tabular}{cccccc}
\hline Índice & DAE & STaS & STaC & Função $\beta(T M s)$ & Função $\beta(T M c)$ \\
\hline $\mathrm{D}$ & 0,77 & 0,88 & 0,89 & 0,83 & 0,90 \\
$\mathrm{C}$ & 0,63 & 0,73 & 0,73 & 0,68 & 0,74 \\
\hline
\end{tabular}

por Willmott et al. (1985) e Camargo \& Sentelhas (1997).

Na Tabela 4, estão os valores do índice de concordância de Willmott (d) e o coeficiente "c", proposto por Camargo \& Sentelhas (1997). Pode ser observado que o maior valor do índice de Willmott foi 0,90 , que correspondeu aos resultados obtidos com a Função $\beta(T M c)$. O valor do coeficiente "c" confirmou o resultado apresentado pelo índice de concordância de Willmott.

\section{CONCLUSÕES}

1. Entre os métodos utilizados, a melhor estimativa do plastocrono do algodoeiro foi obtida com a Função $\beta$, conforme descrita por Wang \& Engel (1998), considerando-se a Temperatura Média Diária Compensada.

2. O método de cálculo utilizado na obtenção da Temperatura Média Diária apresentou influência no resultado da estimativa do plastocrono, nas condições deste experimento.

\section{REFERÊNCIAS}

ANDRADE JÚNIOR, A. S. de et al. Métodos de estimativa da evapotranspiração de referência diária para Parnaíba e Teresina, Piauí. Revista Brasileira de Agrometeorologia, Santa Maria, v. 11, n. 1, p. 63-68, 2003.

ARNOLD, C. Y. The determination and significance of the base temperature in a linear heat unit system. Journal of the American Society for Horticultural Science, Geneva, v. 74, n. 1, p. 430-445, 1959.

AZEVEDO, P. V. de; SILVA, F. D. dos S.; BEZERRA, J. R. C. Zoneamento da época de semeadura do algodoeiro herbáceo no Estado da Paraíba. Revista Brasileira de Agrometeorologia, Santa Maria, v. 12, n. 2, p. 379-386, 2004.

BARBANO, M. T. et al. Temperatura-base e soma térmica para cultivares de ervilha (Pisum sativum L.). Revista Brasileira de Agrometeorologia, Santa Maria, v. 10, n. 1, p. 75-82, 2002. 
CAMARGO, A. P. de; SENTElHAS, P. C. Avaliação do desempenho de diferentes métodos de estimativa da evapotranspiração potencial no Estado de São Paulo, Brasil. Revista Brasileira de Agrometeorologia, Santa Maria, v. 5, n. 1, p. 89-97, 1997.

CAMPELO JÚNIOR, J. H. et al. Efeito da temperatura do ar sobre a abscisão de frutos de mangabeira. In: CONGRESSO BRASILEIRO DE AGROMETEOROLOGIA, 16., 2005, Campinas. Anais... Campinas: SBA/Unicamp, 2005. p. 1-2.

GILMORE, E.; ROGERS, J. S. Heat units as a method of measuring maturity in corn. Agronomy Journal, Madison, v. 50, n. 10, p. 611-615, 1958.

INFELD, J. A.; SILVA, J. B. da; ASSIS, F. N. de. Temperatura-base e graus-dia durante o período vegetativo de três grupos de cultivares de arroz irrigado. Revista Brasileira de Agrometeorologia, Santa Maria, v. 6, n. 2, p. 187-191, 1998.

KHAN, U. Q. Monitoring the growth and development of cotton plants using main stem node counts. Asian Journal of Plant Science, Fasialabad, v. 2, n. 8, p. 593596, 2003.
SENTELHAS, P. C. et al. Temperatura-base e grausdia para cultivares de girassol. Revista Brasileira de Agrometeorologia, Santa Maria, v. 2, n. 1, p. 43-49, 1994.

STRECK, N. A. et al. Estimativa do plastocrono em meloeiro (Cucumis melo L.) cultivado em estufa plástica em diferentes épocas do ano. Ciência Rural, Santa Maria, v. 35 , n. 6 , p. 1275-1280, nov./dez. 2005.

VIATOR, R. P. et al. Predicting cotton boll maturation period using degree days and other climatic factors. Agronomy Journal, Madison, v. 97, n. 2, p. 494-499, mar./ abr. 2005.

WANG, E.; ENGEL, T. Simulation of phenological development of wheat crops. Agricultural Systems, London, v. 58, n. 1, p. 1-24, 1998.

WILLMOTT, C. J. et al. Statistics for the evaluation and comparison of models. Journal of Geophysical Research, Ottawa, v. 90, n. 5, p. 8995-9005, 1985. 